\title{
Symmetry and species segregation in diffusion-limited pair annihilation
}

\author{
H.J. Hilhorst, ${ }^{1}$ M.J. Washenberger, ${ }^{2}$ and U.C. Täuber ${ }^{2}$ \\ ${ }^{1}$ Laboratoire de Physique Théorique, Bâtiment 210, \\ Université de Paris-Sud, 91405 Orsay Cedex, France \\ ${ }^{2}$ Center for Stochastic Processes in Science and Engineering and Department of Physics, \\ Virginia Polytechnic Institute and State University, Blacksburg, Virginia 24061-0435, USA
}

November 20, 2018

\begin{abstract}
We consider a system of $q$ diffusing particle species $A_{1}, A_{2}, \ldots, A_{q}$ that are all equivalent under a symmetry operation. Pairs of particles may annihilate according to $A_{i}+A_{j} \rightarrow 0$ with reaction rates $k_{i j}$ that respect the symmetry, and without self-annihilation $\left(k_{i i}=0\right)$. In spatial dimensions $d>2$ mean-field theory predicts that the total particle density decays as $\rho(t) \sim t^{-1}$, provided the system remains spatially uniform. We determine the conditions on the matrix $k$ under which there exists a critical segregation dimension $d_{\text {seg }}$ below which this uniformity condition is violated; the symmetry between the species is then locally broken. We argue that in those cases the density decay slows down to $\rho(t) \sim t^{-d / d_{\text {seg }}}$ for $2<d<d_{\text {seg }}$. We show that when $d_{\text {seg }}$ exists, its value can be expressed in terms of the ratio of the smallest to the largest eigenvalue of $k$. The existence of a conservation law (as in the special two-species annihilation $A+B \rightarrow 0$ ), although sufficient for segregation, is shown not to be a necessary condition for this phenomenon to occur. We work out specific examples and present Monte Carlo simulations compatible with our analytical results.
\end{abstract}

PACS 05.40.-a, 82.20.-w 


\section{Introduction}

In nonequilibrium statistical mechanics there exist two annihilation reactions that have become prominent model systems. These are the pair annihilation processes $A+A \rightarrow 0$ and $A+B \rightarrow 0$. Interest in fluctuation effects on the first reaction process dates back to the seminal work by Smoluchowski [1, and in more recent times at least to Bramson and Griffeath [2]; and for the second process fluctuations were first studied by Ovchinnikov and Zeldovich [3]. The final states of both processes are trivial: All particles (save perhaps a single one) disappear in the case of $A+A \rightarrow 0$, and only the excess number of particles of the initial majority species is left in the case of $A+B \rightarrow 0$. The nature of the approach to the final state is, however, nontrivial and has been the subject of many investigations.

The $A+A \rightarrow 0$ problem applies, for example, to domain walls in a one-dimensional system like in the zero-temperature Ising model 44. Its asymptotic behavior is the same as that of the diffusion-limited coagulation process $A+A \rightarrow A$ [5]. The ensuing asymptotic density decay $\sim t^{-1 / 2}$ has been experimentally observed (in an intermediate time window) in the fusion kinetics of laser-induced excitons in quasi one-dimensional $\mathrm{N}\left(\mathrm{CH}_{3}\right)_{4} \mathrm{MnCl}_{3}$ (TMMC) polymer chains [6].

The study of the two-species annihilation reaction $A+B \rightarrow 0$ was motivated originally by the cosmological problem of matter-antimatter annihilation. Ovchinnikov and Zeldovich [3], and independently a few years later Toussaint and Wilczek [7, asked whether in such a simple annihilation model it would be possible that locally in space only, say, matter would be left. The answer rapidly turned out to be positive: The $A+B \rightarrow 0$ system exhibits the phenomenon of species "segregation", that is, the emergence of ever growing single-species domains (either $A$ or $B$ ). As a result, after a short initial period, annihilation takes place only in "reaction zones" where the domains border, and the decay of the total density is slowed down markedly. Indeed, a non-classical decay $\sim t^{-3 / 4}$ has been experimentally confirmed in three dimensions in a calcium / fluorophore reaction system 8 . The condition for segregation to occur is that the spatial dimension $d$ be less than the critical segregation dimension $d_{\text {seg }}$, which in the case of $A+B \rightarrow 0$ is equal to $d_{\text {seg }}=4$. Early work on this two-species problem was done by Kang and Redner 9]; clever heuristic reasoning and numerical work on the spatial structure of the domains is due to Leyvraz and Redner [10; and rigorous results were derived by Bramson and Lebowitz [11.

The renormalization group approach to the reaction $A+A \rightarrow 0$ was pursued by Peliti [5] and Lee [12, and to the process $A+B \rightarrow 0$ by Lee and Cardy [13], and Oerding [14]. The strong interest in these two reactions has sparked off research on many related reactiondiffusion problems by various techniques. Here we will refer only to general introductions and overviews, e.g. Refs. [15]-[27].

In 1986 Ben-Avraham and Redner [28], crediting Kang, introduced a $q$-species pair annihilation problem that interpolates between these two models, and hence puts them in a common perspective. They considered a system of $q$ distinct particle species $A_{1}, A_{2}, \ldots, A_{q}$, all propagating with the same difffusion constant, and reacting according to

$$
A_{i}+A_{j} \rightarrow 0 \quad(1 \leq i<j \leq q)
$$

with a single fixed rate. For $q=2$ and $q=\infty$ this $q$-species Mutual Annihilation Model $(q$-MAM) reduces to the well-understood paradigmatic cases $A+B \rightarrow 0$ and $A+A \rightarrow$ 
0, respectively. (For the latter case, note that the probability of identical species to meet becomes vanishingly small in the limit of $q \rightarrow \infty$; hence any particle encounter will lead to a reaction, and the distinction of different species becomes meaningless.) The general situation has recently been studied analytically and by Monte Carlo simulations in Refs. 29, 30, 31.

For equal initial densities of all species the asymptotic decay with time $t$ of the total particle density $\rho(t)$ follows a power law $\sim t^{-\alpha}$. In Table I we summarize the values of the decay exponent $\alpha$, known or believed to be exact, as a function of $q$ and of the spatial dimension $d$ [29, 30, 31]. We also indicate whether or not the density decay is accompanied by particle species segregation. The results for $d=1$ and $q=3,4, \ldots$ are based on the exact solution of a modified model argued by the authors of Refs. [29, 31] to be equivalent to the one-dimensional $q$-MAM in the large-time limit.

\begin{tabular}{||c|c|c|c||}
\hline & $\begin{array}{c}q=2 \\
A+B \rightarrow 0\end{array}$ & $q=3,4, \ldots$ & $\begin{array}{c}q=\infty \\
A+A \rightarrow 0\end{array}$ \\
\hline$d \geq 4$ & 1 & 1 & 1 \\
\hline $2<d<4$ & & & \\
\hline \multicolumn{1}{|c|}{$d / 4(*)$} & $1(\log )$ & $1(\log )$ \\
\cline { 1 - 2 } \cline { 4 - 4 } & & & $d / 2$ \\
\hline $1<d<2$ & & & $1 / 2$ \\
\hline$d=1$ & $1 / 4(*)$ & $(q-1) / 2 q(*)$ & \\
\hline
\end{tabular}

Table I. The density exponent $\alpha$ of the asymptotic decay law $\rho(t) \sim t^{-\alpha}$ for the total partical density in the $q$-species Mutual Annihilation Model, as a function of the number of species $q$ and the spatial dimension $d$. An asterisk $(*)$ indicates that segregation occurs and $(\log )$ signifies the appearance of logarithmic corrections at the upper critical dimension $d_{c}=2$.

The $q$-species MAM is characterized by an upper critical dimension $d_{c}=2$, below which mean-field theory breaks down and renormalization is needed. Physically, in dimensions $d \leq 2$ the recurrence properties of random walkers lead to depletion zones about each surviving particle, and to particle anticorrelations induced by the annihilation kinetics. A systematic renormalization group treatment, wherein the spatial dimension $d$ can be treated as a continuous variable, has only been carried through for the cases $q=\infty$ (equivalent to the reaction $A+A \rightarrow 0$ ) [5], and for $q=2$ (the two-species reaction $A+B \rightarrow 0$ ) [13, 14, which explains the empty entries in the center of Table I.

The derivation of the decay laws listed in Table I relies heavily on the full permutational symmetry of the particle species in the $q$-MAM. It is therefore natural to ask what happens to these asymptotic results when the symmetry is lowered. A case of lower symmetry occurs, for example, when the particle species are ordered cyclically and annihilation, with rate $k_{1}$, is possible only between particles of two neighboring species along the cycle. The reaction constants are then given by

$$
k_{i j}=k_{1}\left[\delta_{(i-j) \bmod q, 1}+\delta_{(i-j) \bmod q, q-1}\right] \quad(1 \leq i, j \leq q) .
$$

We will refer to this particular system as the $q$-species Cyclic Annihilation Model ( $q$ CAM). Other examples that display lower than permutational symmetry may easily be 
constructed.

In this work we develop, within the mean-field framework (i.e., in dimensions $d>2$ ), a general method for finding the exponent of the total density decay law $\rho(t) \sim t^{-\alpha}$, and determining whether or not segregation occurs in a $q$-species model where the reaction rates $k_{i j}$ lower the full permutational symmetry.

In Sec. 2 we present our model, which is fully defined by the $q \times q$ matrix $k$ of annihilation rates. In Sec. 3 we study how the particle numbers of the individual species fluctuate around their average densities at any given time. In Sec. 4 we show, by comparing the average decay law with the fluctuations around it, how the reactions come to an end in a finite $d$-dimensional volume $L^{d}$, and we determine the particle densities in the final state. In Sec. 5 we treat the full $d$-dimensional space as built up from blocks of size $L^{d}$ that do not communicate for times less than the diffusion time, which is of order $L^{2}$. This allows us to derive our general results concerning the occurrence of segregation and the exponents in the density decay law. In Sec. 6 we consider a specific example which, by means of a suitable parameter $\lambda$, interpolates between the $q$-CAM (for $\lambda=0$ ) and the $q$-MAM (for $\lambda=1$ ). In Sec. 7 we present Monte Carlo simulations which, although preliminary and of limited statistical accuracy, are compatible with our analytical findings. In Sec. 8 we comment on an alternative approach, namely via rate equations with an additional particle diffusion term, before we conclude in Sec. 9.

Our considerations allow us to answer another question as well: In the two-species $A+B \rightarrow 0$ system the appearance of segregation is usually explained heuristically through arguments invoking the local conservation of the difference between the number of $A$ and $B$ particles. This conservation law thus seemed to be at the origin of the segregation phenomenon. However, the discovery 29] that the one-dimensional MAM exhibits segregation for all $q<\infty$, even though it is not subject to any conservation law, cast doubt upon this apparent direct link between conservation laws and segregation. This doubt was subsequently reenforced by a heuristic argument according to which the MAM should exhibit segregation in the entire region of the $q$ - $d$ plane delimited by $1<q<3$ and $2<d<4 /(q-1)$ 31. (The only weakness of this argument is that the region concerned by it does not contain any points with integer "physical" $q$ and $d$, except for $q=2$, where the conservation law applies.) This present work clarifies this issue: We show that the existence of a conservation law, although a sufficient condition for segregation in dimensions $d<4$, does not constitute a necessary condition: Segregation may in fact occur in the absence of any conservation law.

\section{A general pair annihilation system}

We consider a system of $q$ distinct diffusing particle species $A_{1}, A_{2}, \ldots, A_{q}$ subject to the pair annihilation reactions

$$
A_{i}+A_{j} \stackrel{k_{i j}}{\longrightarrow} 0 \quad(1 \leq i \neq j \leq q) .
$$

Here the $k_{i j}=k_{j i}$ represent the reaction rates per unit of density; they are constrained only by the requirements that they be non-negative and that there exist a symmetry operation under which all particle species are equivalent. We will set $k_{i i}=0$, implying 
that no self-annihilation is possible. Without loss of generality we may consider the matrix $k$ to be irreducible, i.e., the system of reactants cannot be decomposed into mutually noninteracting subsystems. The $q$-species MAM and the $q$-species CAM discussed in the Introduction are obtained as special cases when all reaction rates in Eq. (3) are equal and when they are given by Eq. (2), respectively.

We shall therefore proceed to analyze the mean-field behavior of the system (3) for a general non-negative traceless symmetric matrix $k$. Our focus will be on the power law for the density decay, $\rho(t) \sim t^{-\alpha}$, and on the segregation properties of this system. Straightforward scaling analysis tells us that for pair annihilation processes such as considered here, very generally mean-field theory is applicable in spatial dimensions $d>d_{c}=2$, and, likely with logarithmic corrections, at the upper critical dimension $d_{c}=2$. We shall operate entirely at the mean-field level, and hence our results will concern dimensions $d \geq 2$. In our discussion we will briefly touch upon implications for lower dimensions $d<2$.

Note that the system of reactions (3) may be visualized as a graph $\mathcal{G}$ with $q$ vertices representing the $q$ species, in which bonds carrying weights $k_{i j}$ connect the vertices of pairs of species that may react with each other. At certain points in our discussion it will be convenient to refer to this graph representation.

\section{Mean-field theory and fluctuations}

\subsection{Averages}

Let the stochastic variable $N_{i}(t)$ denote the particle number of species $A_{i}$ present at time $t$ in a system of volume $L^{d}$, and let $n_{i}(t)=\left\langle N_{i}(t)\right\rangle / L^{d}$ be the average density of the $A_{i}$ for $i=1,2, \ldots, q$. Here $\langle\ldots\rangle$ is an average with respect to (i) the initial distribution of the $N_{i}$ at time $t=0$ and (ii) all realizations of the stochastic time evolution. In order to express the mean-field rate equations $\mathrm{d} n_{i} / \mathrm{d} t=-\sum_{j} k_{i j} n_{i} n_{j}$ in terms of dimensionless variables, we set $k_{0}=\sum_{j} k_{i j}$ (which because of the symmetry between all species is independent of $i$ ) and $n_{0}=q^{-1} \sum_{i} n_{i}(0)$. We furthermore define the quantities $\rho_{i}(t)=n_{i}(t) / n_{0}, \kappa_{i j}=k_{i j} / k_{0}$, and $\tau=n_{0} k_{0} t$. The mean-field equations then become

$$
\frac{\mathrm{d} \rho_{i}}{\mathrm{~d} \tau}=-\sum_{j} \kappa_{i j} \rho_{i} \rho_{j},
$$

now with the normalization $\sum_{j} \kappa_{i j}=1$. If all initial densities $n_{i}(0)=n_{0}$ and hence $\rho_{i}(0)=1$ for all $i$, then the solution of Eq. (4) reads

$$
\rho_{i}(\tau)=\rho(\tau)=\frac{1}{1+\tau}
$$

which tends to zero as a power law with decay exponent $\alpha=1$. For unequal initial densities the asymptotic decay will generically be exponential (readily generalized to stretched exponential for $d \leq 2$ as a consequence of the reaction rate renormalization [9, 11, 27]), with one or several of the species tending toward a positive limit density $\rho_{i}(\infty)$; this case will not be considered any further below. 


\section{$3.2 \quad$ Fluctuations}

In principle, the fluctuations around this mean-field average are encoded in the master equation for the probability distribution $P\left(N_{1}, N_{2}, \ldots, N_{q} ; t\right)$,

$$
\frac{\mathrm{d} P}{\mathrm{~d} t}=\sum_{1 \leq i<j \leq q} \frac{k_{i j}}{L^{d}}\left[\left(N_{i}+1\right)\left(N_{j}+1\right) P\left(\ldots, N_{i}+1, N_{j}+1, \ldots\right)-N_{i} N_{j} P\left(\ldots, N_{i}, N_{j} \ldots\right)\right] .
$$

A convenient means to extract them proceeds via van Kampen's $\Omega$-expansion [32, as was demonstrated for the $q$-MAM by Ben-Avraham and Redner [28, and more recently for a cyclic three-species trapping reaction by Ben-Naim and Krapivsky [33], and for a zero-dimensional population dynamics model by Newman and McKane 34.

Anticipating that the fluctuations should be of the order of the square root of the total particle number $\sum_{i=1}^{q} N_{i}$, we set

$$
N_{i}(t)=n_{0} L^{d} \rho(\tau)+\left(n_{0} L^{d}\right)^{1 / 2} \gamma_{i}(\tau)
$$

where, due to previous definitions, $\left\langle\gamma_{i}(0)\right\rangle=0$, and transform the probability distribution $P$ on the extensive variables $N_{i}$ into an equivalent one, to be called $F$, on the intensive variables $\gamma_{i}$, according to

$$
P\left(N_{1}, N_{2}, \ldots, N_{q} ; t\right)=\left(n_{0} L^{d}\right)^{q / 2} F\left(\gamma_{1}, \gamma_{2}, \ldots, \gamma_{q} ; \tau\right) .
$$

Expanding the master equation (6) for $P$ to second order in powers of $\left(n_{0} L^{d}\right)^{-1 / 2}$, as in Refs. 28, 33], and [34, and exploiting the rate equations (44) for $\rho_{i}(\tau)=\rho(\tau)$ then yields a Fokker-Planck equation with time-dependent coefficients,

$$
\partial_{\tau} F=\sum_{1 \leq i<j \leq q} \kappa_{i j}\left[\rho\left(\partial_{i}+\partial_{j}\right)\left(\gamma_{i}+\gamma_{j}\right)+\frac{1}{2} \rho^{2}\left(\partial_{i}+\partial_{j}\right)^{2}\right] F
$$

where $\partial_{i} \equiv \partial / \partial \gamma_{i}$. This equation is valid provided the second term on the r.h.s. of Eq. (7) remains much smaller than the first one.

\subsection{Equations for the second moments}

From the Fokker-Planck equation (9) we may readily deduce equations for the time evolution of the averages $\left\langle\gamma_{i}\right\rangle$, the variances $\left\langle\gamma_{i}^{2}\right\rangle \equiv \Gamma_{i i}$, and the covariances $\left\langle\gamma_{i} \gamma_{j}\right\rangle \equiv \Gamma_{i j}$. Since $\mathrm{d}\left\langle\gamma_{i}\right\rangle / \mathrm{d} \tau=-\rho\left\langle\gamma_{i}\right\rangle-\rho \sum_{j} \kappa_{i j}\left\langle\gamma_{j}\right\rangle$, and given that the $\left\langle\gamma_{i}\right\rangle$ are zero initially, we see that they vanish for all times. The covariance matrix $\Gamma$ satisfies

$$
\frac{\mathrm{d} \Gamma_{i j}}{\mathrm{~d} \tau}=\rho^{2}\left(\kappa_{i j}+\delta_{i j}\right)-2 \rho \Gamma_{i j}-\rho \sum_{\ell} \kappa_{i \ell} \Gamma_{j \ell}-\rho \sum_{\ell} \kappa_{j \ell} \Gamma_{i \ell} .
$$

Now let $U$ be the real unitary matrix with elements $U_{\mu i}$ that renders $\hat{\kappa}=U \kappa U^{-1}$ diagonal, and denote its eigenvalues by $\hat{\kappa}_{\mu}$. In the same manner, we define $\hat{\Gamma}=U \Gamma U^{-1}$. Upon transforming Eq. (10) to these new variables we obtain

$$
\frac{\mathrm{d} \hat{\Gamma}_{\mu \nu}}{\mathrm{d} \tau}=\left[1+\frac{1}{2}\left(\hat{\kappa}_{\mu}+\hat{\kappa}_{\nu}\right)\right]\left(\rho^{2} \delta_{\mu \nu}-2 \rho \hat{\Gamma}_{\mu \nu}\right) .
$$


Let us suppose that the particle numbers initially have independent and identical fluctuations, i.e., $\Gamma_{i j}(0)=\Gamma_{0} \delta_{i j}$. Then also $\hat{\Gamma}_{\mu \nu}(0)=\Gamma_{0} \delta_{\mu \nu}$, and it follows from Eq. (11) that $\hat{\Gamma}_{\mu \nu}(\tau)=0$ for $\mu \neq \nu$ at all $\tau>0$. The remaining equation of interest is

$$
\frac{\mathrm{d} \hat{\Gamma}_{\mu \mu}}{\mathrm{d} \tau}=\left(1+\hat{\kappa}_{\mu}\right) \rho^{2}-2\left(1+\hat{\kappa}_{\mu}\right) \rho \hat{\Gamma}_{\mu \mu} .
$$

Notice that since $\rho$ depends on time, it is not useful to scale the time $\tau$ in (12) with the factor $1+\hat{\kappa}_{\mu}$. The normalization relation $\sum_{j} \kappa_{i j}=1$ implies that $\hat{\kappa}_{0} \equiv 1$ is an eigenvalue of $\kappa$ with eigenvector $(1,1, \ldots, 1)$. By the Perron-Frobenius theorem, and since the $\hat{\kappa}_{\mu}$ are necessarily real, we have $-1 \leq \hat{\kappa}_{\mu} \leq 1$ for all $\mu$. In case $\hat{\kappa}_{\mu}=-1$, the r.h.s. of Eq. (12) vanishes and the initial fluctuation $\hat{\Gamma}_{\mu \mu}$ does not decay, i.e., an eigenvalue -1 signals the presence of a conservation law.

\subsection{Solution of the moment equations}

The solution of Eq. (12), except when $\hat{\kappa}_{\mu}=-\frac{1}{2}$, is a sum of two power laws,

$$
\hat{\Gamma}_{\mu \mu}(\tau)=\left(\Gamma_{0}-K_{\mu}\right)(1+\tau)^{-2\left(1+\hat{\kappa}_{\mu}\right)}+K_{\mu}(1+\tau)^{-1}
$$

where we have introduced the coefficient $K_{\mu}=\left(1+\hat{\kappa}_{\mu}\right) /\left(1+2 \hat{\kappa}_{\mu}\right)$. Note that the amplitude of (only) the first term in Eq. (13) depends on the initial fluctuation strength $\Gamma_{0}$. Its exponent $2\left(1+\hat{\kappa}_{\mu}\right)$ varies for the different modes $\mu$, which shows that this problem is characterized by a spectrum of power laws. The second term in Eq. (13) is just proportional to the density $\rho(\tau)$ and hence decays as $\tau^{-1}$. Therefore, if $\hat{\kappa}_{\mu}<-\frac{1}{2}$, the first term decays more slowly than the second one (and vice versa for $\hat{\kappa}_{\mu}>-\frac{1}{2}$ ). When it so happens that $\kappa_{\mu}=-\frac{1}{2}$, then Eq. (13) should be replaced with the special logarithmic solution

$$
\hat{\Gamma}_{\mu \mu}(\tau)=\Gamma_{0}(1+\tau)^{-1}+\frac{1}{2}(1+\tau)^{-1} \log (1+\tau) .
$$

The variance of the particle number for species $i$ is given by

$$
\left\langle\Delta N_{i}^{2}(t)\right\rangle=q^{-1} \sum_{i}\left\langle\Delta N_{i}^{2}(t)\right\rangle=n_{0} L^{d} q^{-1} \sum_{i} \Gamma_{i i}(\tau)=n_{0} L^{d} q^{-1} \sum_{\mu} \hat{\Gamma}_{\mu \mu}(\tau) .
$$

Let us denote the algebraically smallest eigenvalue of $\kappa$ by $\hat{\kappa}_{\mathrm{m}}$, its degeneracy by $c_{\mathrm{m}}$, and the corresponding value of the coefficient $K_{\mu}$ by $K_{\mathrm{m}}$. For asymptotically large times we then deduce from Eqs. (13)-(15) the behavior

$$
\Gamma_{i i}(\tau) \simeq\left\{\begin{array}{l}
K \tau^{-1} \\
\frac{1}{2} \tau^{-1} \log \tau \\
c_{\mathrm{m}} q^{-1}\left(\Gamma_{0}-K_{\mathrm{m}}\right) \tau^{-2\left(1+\hat{\kappa}_{\mathrm{m}}\right)}
\end{array} \quad\left(\hat{\kappa}_{\mathrm{m}} \gtreqless-\frac{1}{2}, \quad \tau \rightarrow \infty\right)\right.
$$

with the abbreviation $K=q^{-1} \sum_{\mu} K_{\mu}$. 


\section{Final state in a finite volume}

We are now ready to exploit the results of the preceding sections. We begin by posing the question whether there exists a characteristic time $\tau_{*}(L)=n_{0} k_{0} t_{*}(L)$ at which the particle number $N_{i}$ in the volume $L^{d}$ displays a root mean-square deviation from the average that is of the order of the average itself, i.e.,

$$
\left\langle N_{i}\left(t_{*}\right)\right\rangle \simeq\left\langle\Delta N_{i}^{2}\left(t_{*}\right)\right\rangle^{1 / 2}
$$

Yet the Fokker-Planck equation (9) was derived under the hypothesis of small fluctuations (the second term in Eq. (77) was supposed to small compared to the first one), and hence ceases to be valid for $\tau \gtrsim \tau_{*}(L)$. The issue then is what happens at and beyond this time scale. Fluctuations satisfying (17) indicate that the different particles species have widely varying numbers. As we argued in the Introduction, in this situation the annihilation processes come to an end epxonentially, on the prevailing time scale, through the successive extinction of one or several particle species; this final decay is no longer described by the Fokker-Planck equation (9). In the finite volume $L^{d}$, the final state therefore consists of a collection of particles that are not subject to pair annihilations anymore. If all $\kappa_{i j}>0$ for $i \neq j$, then at most a single species can survive. But if some of the rates $\kappa_{i j}$ vanish, a broader variety of final states is possible (we shall return to this point in Sec. 6). In any of these cases, whereas initially all species were equivalent, the final state has this symmetry broken. In fact, this symmetry breaking may be traced back to the first term in Eq. (13), which includes the amplitude $\Gamma_{0}-K_{\mu}$. Thus, this symmetry breaking is enhanced by initial fluctuations, as represented by $\Gamma_{0}$, yet it actually persists even for the case $\Gamma_{0}=0$, i.e. if we initially take all particle numbers $N_{i}(0)$ to be strictly equal. We shall discuss several examples of such broken symmetry states in Sec. 6.

We turn now to the determination of the characteristic time $\tau_{*}(L)$. Utilizing in Eq. (17) that $\left\langle N_{i}(t)\right\rangle \simeq n_{0} L^{d} \tau^{-1}$ for $\tau \rightarrow \infty$ as well as inserting the asymptotic results of Eqs. (15) and (16), and then solving for $\tau_{*}(L)$, we find

$$
\tau_{*}(L) \simeq\left\{\begin{array}{l}
K^{-1} n_{0} L^{d} \\
2 n_{0} L^{d} / \log \left(n_{0} L^{d}\right) \\
C^{-1}\left(n_{0} L^{d}\right)^{1 /\left|2 \hat{\kappa}_{\mathrm{m}}\right|}
\end{array} \quad\left(\hat{\kappa}_{\mathrm{m}} \gtreqless-\frac{1}{2}, \quad \tau \rightarrow \infty\right),\right.
$$

where $C=\left[c_{\mathrm{m}} q^{-1}\left(\Gamma_{0}-K_{\mathrm{m}}\right)\right]^{1 /\left|2 \hat{\kappa}_{\mathrm{m}}\right|}$. The order of magnitude $N_{*}(L)$ of the particle number for a surviving species will be $N_{*}(L) \sim n_{0} L^{d} \rho\left(\tau_{*}(L)\right) \simeq n_{0} L^{d} / \tau_{*}(L)$, and hence is given by

$$
N_{*}(L) \sim\left\{\begin{array}{l}
K \\
\frac{1}{2} \log \left(n_{0} L^{d}\right) \\
C\left(n_{0} L^{d}\right)^{1-1 /\left|2 \hat{\kappa}_{\mathrm{m}}\right|}
\end{array} \quad\left(\hat{\kappa}_{\mathrm{m}} \gtreqless-\frac{1}{2}, \quad \tau \rightarrow \infty\right) .\right.
$$

When $\hat{\kappa}_{\mathrm{m}}>-\frac{1}{2}$ this surviving number is of order unity, but when $\hat{\kappa}_{\mathrm{m}}=-\frac{1}{2}$ it grows logarithmically with the volume; and when $\hat{\kappa}_{\mathrm{m}}<-\frac{1}{2}$ it grows with a positive power of $L$. 


\section{Segregation in an infinite volume}

We now consider an infinite volume in which the $q$ annihilating species propagate with (uniform) diffusion constant $D$, and apply a heuristic analysis: We imagine this infinite volume divided into hypercubic subvolumes of size $L^{d}$. For times less than the characteristic diffusion time, i.e., for $\tau \lesssim \tau_{\text {diff }}(L) \sim L^{2} / D$, the subvolumes can be treated as effectively independent, and hence the results of Eqs. (18) and (19) apply to each of them. We will discuss the four distinct cases separately.

(i) Case $-\frac{1}{2}<\hat{\kappa}_{\mathrm{m}} \leq 1$. From Eq. (19) we infer in this situation that in an isolated volume the particle number fluctuations become of the order of the average itself only at times when the total particle numbers $N_{*}(L) \sim K$ have decreased to order unity themselves. In any case, in dimensions $d>2$ the time scale at which this happens, $\tau_{*}(L) \sim L^{d}$, is much larger than the diffusion time $\tau_{\text {diff }}(L) \sim L^{2}$. Hence in an infinite system the subvolumes begin to mix diffusively long before the fluctuations of their particle numbers become of the order of the averages. This is tantamount to stating that for $\hat{\kappa}_{\mathrm{m}}>-\frac{1}{2}$ there is no particle segregation.

(ii) Case $-1<\hat{\kappa}_{\mathrm{m}}<-\frac{1}{2}$. In this case Eq. (18) states that the fluctuations become of the order of the average value $\rho(\tau)$ at a characteristic time $\tau_{*}(L) \sim L^{d /\left|2 \hat{\kappa}_{\mathrm{m}}\right|}$, and in an isolated volume the annihilation processes will come to an end on this same time scale. Similarly in an infinite system, subject to the condition that

$$
\tau_{*}(L) \ll \tau_{\text {diff }}(L)
$$

each subvolume of size $L^{d}$ will reach a quasi-final state, to be referred to as a "domain", and further reactions are possible only at the "reaction zones" separating the domains. The quasi-final state in each subvolume is reached independently of its neighbors, and the emergence of these different disjoint quasi-final states constitutes the phenomenon of segregation into domains. The condition (20) implies that segregation occurs in dimensions below a critical segregation dimension $d_{\text {seg }}$

$$
d_{\mathrm{seg}}=4\left|\hat{\kappa}_{\mathrm{m}}\right| \text {. }
$$

It therefore appears that in the present case the critical segregation dimension for diffusionlimited multi-species pair annihilation processes is always constrained by the interval $2<$ $d_{\text {seg }}<4$. The dimensionless particle density in a given domain will be of the order $\rho_{*}(L)=$ $N_{*}(L) /\left(n_{0} L^{d}\right)$ and cannot decrease further until diffusion between neighboring domains permits new reactions to take place. Hence we conclude that at any time $\tau$ the particle density in an infinite system equals $\rho_{*}\left(L_{\text {diff }}(t)\right)$, where $L_{\text {diff }}(t) \sim(D t)^{1 / 2}=\left(D \tau / n_{0} k_{0}\right)^{1 / 2}$. By combining these relations we find that the density decays asymptotically as

$$
\rho(\tau) \sim\left(C^{\prime} \tau\right)^{-d / d_{\text {seg }}} \quad\left(2<d<d_{\text {seg }}\right),
$$

where $C^{\prime}=\left[c_{\mathrm{m}} q^{-1}\left(\Gamma_{0}-K_{\mathrm{m}}\right)\right]^{-2 / d} n_{0}^{2 / d-1} D / k_{0}$ is a dimensionless constant. Thus we obtain $\alpha \equiv d / d_{\text {seg }}$ for $2<d<d_{\text {seg }}$.

(iii) Case $\hat{\kappa}_{\mathrm{m}}=-\frac{1}{2}$. In this case $d_{\text {seg }}=2$, which is also the upper critical dimension $d_{c}$ where mean-field theory is only marginally applicable. Nevertheless, upon following the 
same reasoning as above we find the asymptotic density decay law

$$
\rho(\tau) \sim\left(k_{0} / 2 D\right) \tau^{-1} \log \left(D \tau / k_{0}\right) \quad\left(d=d_{\text {seg }}=d_{c}=2\right)
$$

in two dimensions. Based on renormalization group arguments and Monte Carlo simulations, this result was predicted in particular for the 3-MAM [29, 30, 31, for which indeed $\hat{\kappa}_{\mathrm{m}}=-\frac{1}{2}$ (see Subsec. 6.1 below). It is remarkable that the logarithmic corrections (23) take the same form as quite generically predicted for pair annihilation reactions in two dimensions as consequence of the required reaction rate renormalizations induced by the appearance of depletion zones in low dimensions. Although segregation effects are very difficult to capture within the framework of renormalized field theory (compare the discussion in Ref. 31]), we therefore hypothesize, but cannot prove, that no additional logarithmic factors beyond those exhibited in Eq. (23) appear in the general case.

(iv) Case $\hat{\kappa}_{\mathrm{m}}=-1$. This special case, for which $d_{\text {seg }}=4$, occurs when the graph $\mathcal{G}$ is bipartite, i.e., when it is comprised of two subsets of vertices such that all bonds are between vertices of different subsets. Since these subsets are necessarily equivalent under symmetry, this obviously requires that $q$ be even, and we may relabel the sets to $\{1,2, \ldots, q / 2\}$ and $\{q / 2+1, q / 2+2, \ldots, q\}$. The particle numbers then obey the local conservation law

$$
\sum_{i=1}^{q / 2} N_{i}(t)-\sum_{i=q / 2+1}^{q} N_{i}(t)=\text { constant },
$$

and it is readily verified that $\kappa$ has an eigenvector $(1, \ldots, 1,-1, \ldots,-1)$ with eigenvalue -1 . For $\hat{\kappa}_{\mathrm{m}}=-1$ we moreover infer from Eq. (13) that $\hat{\Gamma}_{\mu \mu}(\tau)=\Gamma_{0}$, which establishes that as a direct consequence of the conservation law the initial fluctuations in the total particle numbers of the two subsets do not relax. Examples where this happens are the 2-MAM (the two-species pair annihilation reaction $A+B \rightarrow 0$ ) and the $q$-CAM with even $q$ (see Subsec. 6.2).

\section{An explicit example}

We proceed to investigate a specific $q$-species pair annihilation system that depends on a parameter $\lambda$, interpolating for $0 \leq \lambda \leq 1$ between the $q$-CAM and the $q$-MAM that were defined in the Introduction.

We imagine the $q$ species arranged in a cycle. The annihilation rate is set equal to $k_{1}$ for any pair of species that are nearest neighbors along the cycle, and to $k_{2}$ for any other pair. Then the ratio $\lambda \equiv k_{2} / k_{1}$ is the only intervening parameter in the normalized matrix $\kappa$. Using the abbreviation $\mathcal{N}_{q \lambda}=1+\frac{1}{2}(q-3) \lambda$, one has

$$
\kappa_{i j}=\left[\lambda+(1-\lambda)\left(\delta_{(i-j) \bmod q, 1}+\delta_{(i-j) \bmod q, q-1}\right)\right] / 2 \mathcal{N}_{q \lambda} \quad(i \neq j)
$$

and $\kappa_{i i}=0$. For $0 \leq \lambda<1$ this model is symmetric only under cyclic translation of the species, and the special case $\lambda=0$ yields the $q$-CAM. For $\lambda=1$ the model displays full permutation symmetry and we recover the $q$-MAM. 
It is straightforward to diagonalize $\kappa$ by Fourier transforming in the variable $i-j$, and hence the mode labels $\mu, \nu, \ldots$ belong to the set of wavenumbers $p \in\{2 \pi n / q\}$ with $n=0,1, \ldots, q-1$. The corresponding eigenvalues are

$$
\hat{\kappa}_{p}=q^{-1} \sum_{i, j} \kappa_{i j} \mathrm{e}^{\mathrm{i} p(i-j)}=\left[\frac{1}{2} \lambda\left(q \delta_{p 0}-1\right)+(1-\lambda) \cos p\right] / \mathcal{N}_{q \lambda} .
$$

We shall first discuss the two limiting models, the MAM and the CAM, whose segregation properties turn out to be quite different. Next, our study as a function of $\lambda$ will characterize the crossover between these two cases, thereby shedding light on the nature of the segregation mechanism.

\subsection{Mutual Annihilation Model (MAM)}

The parameter value $\lambda=1$ defines the Mutual Annihilation Model ( $q$-MAM). In this case the graph $\mathcal{G}$ with $q$ vertices is fully connected and every bond carries the same weight. This model, introduced in Ref. [28, has been extensively studied in Refs. 29, 30, 31. We may reproduce the known results in dimensions $d \geq 2$ as follows. Eq. (26) shows that for $\lambda=1$ the matrix $\kappa$ has a single eigenvalue 1 (namely for the mode with $p=0$ ) and a $(q-1)$-fold degenerate eigenvalue $\hat{\kappa}_{\mathrm{m}}=-1 /(q-1)$ (which occurs for all $p \neq 0$ ). In the two-species model with $q=2$, this latter eigenvalue equals -1 , whence we encounter the special case (iv) of Sec. 5. There is a conservation law, we find $d_{\text {seg }}=4$, and for $d<d_{\text {seg }}$ the density decays as $\rho(\tau) \sim \tau^{-d / 4}$ [9, 11, 13, 31].

For $2<q<3$, the eigenvalue $\hat{\kappa}_{\mathrm{m}}=-1 /(q-1)$ lies in the interval $\left[-1,-\frac{1}{2}\right]$, whence we are confronted with case (ii) of Sec. 5. According to Eq. (21), there then exists a $q$-dependent segregation dimension

$$
d_{\mathrm{seg}}(q)=4 /(q-1)
$$

a relation first derived in Ref. 31. Although this case does not encompass any integer values of $q$, it does suggest that segregation may occur even in the absence of a conservation law. Such a scenario will indeed be confirmed below.

The marginal value $q=3$ corresponds to the special case (iii) of Sec. 5. Indeed, it was concluded in Refs. [29, 31] on the basis of renormalization group arguments that in two dimensions the total density in the 3-MAM decays as $\rho(\tau) \sim \tau^{-1} \log \tau$, in agreement with Eq. (23). For $q>3$ we obtain $d_{\text {seg }}(q)<2$, and our present theory is not applicable anymore. The analytical and numerical studies reported in Refs. [29, 30, 31] however yield species segregation in one dimension, with a $q$-dependent density decay exponent $\alpha(q)=(q-1) / 2 q$.

\subsection{Cyclic Annihilation Model (CAM)}

For $\lambda=0$ the cyclic graph $\mathcal{G}$ only contains nearest-neighbor bonds, i.e., a particle of a given species can annihilate only with particles of one of its two neighboring species. This model has been called the Cyclic Annihilation Model or q-CAM. In this case the eigenvalues given in Eq. (26) reduce to $\hat{\kappa}_{p}=\cos p$. For even $q$, the mode with $p=\pi$ yields 
the smallest eigenvalue $\hat{\kappa}_{\mathrm{m}}=-1$. But for odd $q$ the smallest eigenvalue is acquired for $p=\pi\left(1 \pm q^{-1}\right)$, namely $\hat{\kappa}_{\mathrm{m}}=-\cos \frac{\pi}{q}$. It then follows from Eq. (21) that

$$
d_{\mathrm{seg}}=\left\{\begin{array}{ll}
4 & (q=2,4,6, \ldots) \\
4 \cos \frac{\pi}{q} & (q=3,5,7, \ldots)
\end{array} .\right.
$$

For $q=2,3,4$ these results for $d_{\text {seg }}(q)$ were previously known, since in these cases the CAM actually coincides with a MAM: the 3-CAM coincides with the 3-MAM, and the 2-CAM and 4-CAM are both equivalent to the 2-MAM [29, 31]. The first novel case is therefore $q=5$, for which Eq. (28) gives

$$
d_{\mathrm{seg}}(5)=1+\sqrt{5}=3.236 \ldots
$$

It follows that the 5-CAM exhibits segregation in spatial dimensions $d=3$ and $d=2$, and Eq. (22) implies that $\rho(\tau) \sim \tau^{-\alpha}$ with

$$
\alpha=\left\{\begin{array}{ll}
\frac{1}{2}(\sqrt{5}-1)=0.618 \ldots & (d=2) \\
\frac{3}{4}(\sqrt{5}-1)=0.927 \ldots & (d=3)
\end{array} \quad(q=5) .\right.
$$

(Curiously, the decay exponent in two dimensions is just the golden mean.) In simulations, aside from the observation of species segregation, direct measurement of the exponent values $\alpha$ provides a straightforward means to verify the present theory (see Sec. 7 below).

The domain structure in the case of the $q$-CAM needs to be discussed. Whereas the MAM is necessarily characterized by single-species domains, this is no longer true for the CAM. Let us first consider $q=5$. For the 5-CAM one can certainly conceive the possibility of single-species domains. Any given particle species, however, does not react with two other species, and hence is able to coexist with either of those. It appears evident, therefore, that a single-species domain is not stable against penetration by either of the two species with which its particles cannot annihilate. Thus we must suppose that a typical segregated domain will always contain two species, say $\ell$ and $\ell^{\prime}$, out of the five, viz. any of the cyclically equivalent combinations $\left\{\ell \ell^{\prime}\right\}=\{13\},\{24\},\{35\},\{41\},\{52\}$. These domains are "stable against penetration", in the following sense: Any particle of a different species intruding into such a domain would annihilate with at least one of the two domain species.

For $q \geq 6$ new questions appear that we will not fully address here. As for $q=5$, we may list the subsets of particles that can coexist in a domain where no interactions take place anymore. There are two subsets with three species, $\{135\}$ and $\{246\}$, and three subsets with only two species, namely $\{14\},\{25\}$, and $\{36\}$. All these five subsets are "impenetrable" to an intruder species. It is therefore an interesting issue which type of domains are going to be formed in the segregation process.

One important conclusion from the $q$-CAM is that for $q=5,7, \ldots$ we encounter "physical" situations ( $q$ and $d$ are integers), where, in spite of the absence of a conservation law, species segregation into domains emerges. Note that the segregation mechanism does not even require initial fluctuations in the species numbers: Even if initially absent, such fluctuations are dynamically generated by the annihilation reactions, and they decay more slowly than the average particle numbers themselves. 


\subsection{Interpolating model}

We now allow an arbitrary value for $\lambda$ in the interval $[0,1]$, i.e., we consider the more general interpolating model. For even $q$, Eq. (26) tells us that the eigenvalue $\hat{\kappa}_{p}$ assumes its minimum for $p=\pi$; for odd $q$ one may verify that, just as for $\lambda=1$, it becomes minimal for $p$ as close as possible to $\pi$, that is, for $p=\pi\left(1 \pm q^{-1}\right)$. Upon substituting these minimal eigenvalues in Eq. (21), we obtain

$$
d_{\mathrm{seg}}(q)= \begin{cases}(4-2 \lambda) / \mathcal{N}_{q \lambda} & q \text { even } \\ {\left[2 \lambda+4(1-\lambda) \cos \frac{\pi}{q}\right] / \mathcal{N}_{q \lambda}} & q \text { odd }\end{cases}
$$

For $\lambda=0,1$, this expression reduces to the limiting values found above. It is consistent with our mean-field assumptions only as long as it leads to a $d_{\operatorname{seg}}(q) \geq 2$. That is easily seen to imply that at given $\lambda$, it is meaningful only for $q$ below a maximum value $q_{c}(\lambda)$; for $q$ above that value, there is no segregation in any $d \geq 2$.

\section{Simulations}

We have performed Monte Carlo simulations in one, two, and three dimensions for the $q$-CAM with $q=2,3,4,5$ with the goal to test the theoretical predictions for the decay exponent $\alpha$. For more particle species, our computing resources unfortunately cannot provide sufficiently reliable data statistics.

The simulation algorithm proceeds as described in Ref. [31]: Starting from an initially random distribution of particles on a $d$-dimensional $(d=1,2,3)$ hypercubic lattice with periodic boundary conditions, the system is evolved as follows: A particle is randomly picked. Next, one of its $2 d$ nearest-neighbor sites is selected randomly; if it is occupied by a particle of a different species, both particles are removed, otherwise the particle hops to the empty site. The Monte Carlo time is scaled with the total number of particles present (asynchronous time update).

Beginning with the data in three dimensions, obtained from simulations on a $100 \times$ $100 \times 100$ cubic lattice by averaging over 20 runs with random initial conditions, we plot the effective decay exponent

$$
\alpha(t)=-\frac{\mathrm{d} \ln \rho(t)}{\mathrm{d} \ln t}
$$

in Fig. 1. In $d=3$, naturally our statistics is worst, and the data cease to be reliable at around $t \approx 600$. The results for even and odd $q$ are clearly distinct; and as anticipated, the processes for $q=2$ and $q=4$ are equivalent. Whereas Eqs. (28) and (22) predict $\alpha=3 / 4$ asymptotically for $q$ even, the data show that the effective exponent $\alpha(t)$ rather reaches a plateau at $\sim 0.88$. As discussed in Ref. [31], one should however expect the asymptotic decay law to be somewhat masked by the mean-field behavior $\sim t^{-1}$, which could explain the combined effective decay exponent closer to 1 . Indeed, we found the same deviation from the asymptotic value 0.75 in our simulations for the 2-MAM (see Fig. 8 in Ref. [31]). The data for odd $q$ are similarly plagued by crossover effects. For

$q=3$, for which the MAM and CAM are equivalent, we see a slow convergence towards 


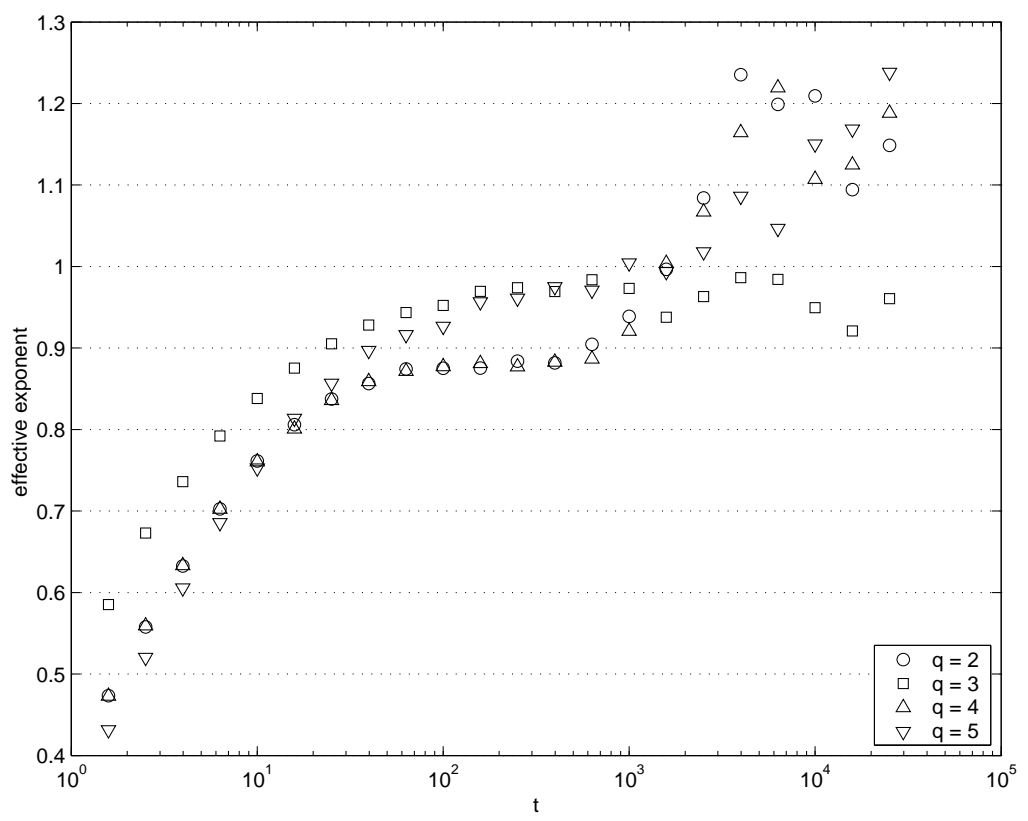

Figure 1: Effective decay exponent $\alpha(t)$ for the three-dimensional $q$-CAM with $q=2,3,4$, and 5 (random initial conditions, equal initial particle numbers for each species). The data were obtained on a $100 \times 100 \times 100$ cubic lattice, averaged over 20 runs.

$\alpha=1$. The data for $q=5$ might indicate that $\alpha(t)$ reaches a plateau at a smaller value, in line with the prediction $\alpha \approx 0.93$ of Eq. (30), but the deteriorating statistics preclude a definite conclusion.

Our two-dimensional results, from 20 runs on a $1000 \times 1000$ square lattice, are depicted in Fig. 2 The data become unreliable at about $t \approx 1000$. Once more, the $q$-CAM for $q=2$ and $q=4$ are seen to be equivalent, with $\alpha(t)$ settling towards the asymptotic value $1 / 2$, albeit masked again by the competing mean-field power law, just as for the 2-MAM (see Fig. 6 in Ref. [31]). Yet now the runs for $q=3$ and $q=5$ yield manifestly different power laws. In the three-species CAM, the effective exponent is still changing in the time window accessible to our simulations, running towards $\alpha=1$, perhaps with logarithmic corrections as predicted by Eq. (23). For $q=5$, however, we find a plateau value $\sim 0.71$, perhaps with a slowly decreasing tendency. This may be interpreted as a combination of the predicted asymptotic decay law (30) with the mean-field result $\sim t^{-1}$.

The simulation data in $d=1$, from averaging over again 20 runs on $10^{5}$ lattice sites, are reliable at least up to $t \approx 10^{4}$, and again confirm the equivalence of the $q$-CAM with even $q$. For both $q=2$ and $q=4$ we obtain a slow but definite approach towards the predicted $\alpha=1 / 4$, as for the 2-MAM (see Fig. 2 in Ref. [31]). For the 3-CAM, equivalent to the 3-MAM, we find the expected slow convergence towards $\alpha=1 / 3$ from above [29, 30, 31]. Remarkably, the data for $q=5$, for which no analytical prediction is available in one dimension, appear to reach $\alpha(t) \approx 0.33$ as well, but faster and from below. This is remarkably close to what Eq. (22) would predict if we applied it (without justification) in dimension $d=1$, namely $\alpha=1 / d_{\mathrm{seg}}(5)=0.309 \ldots$, with the segregation 


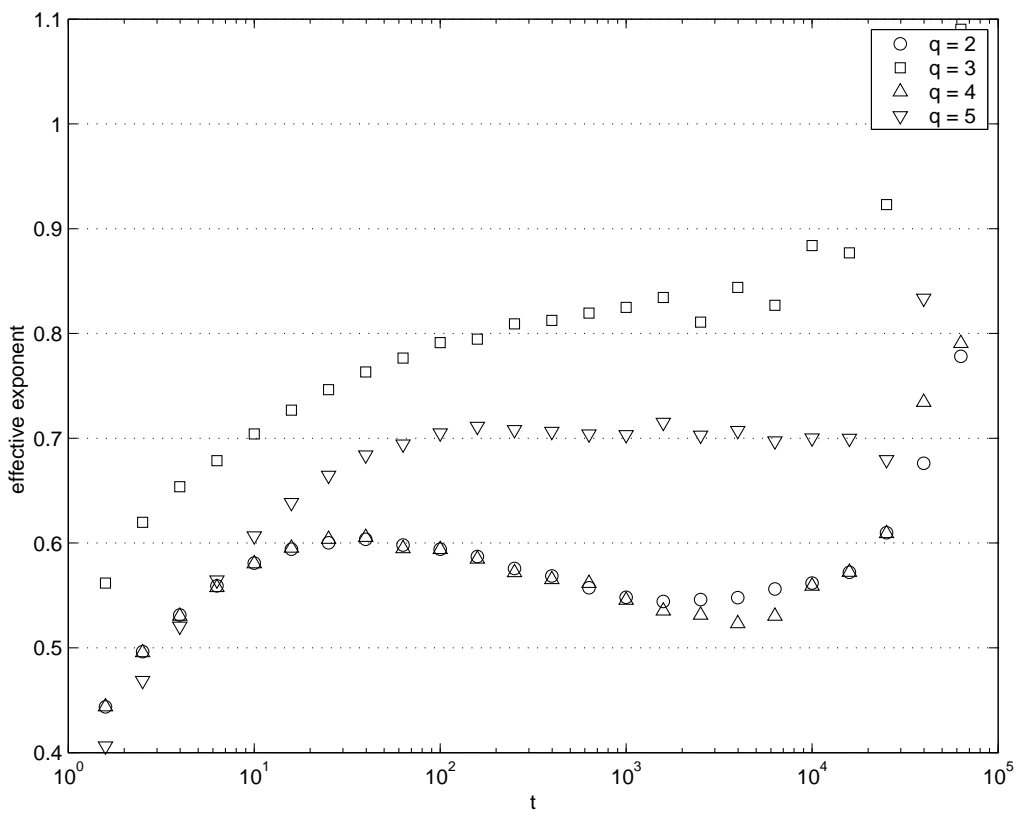

Figure 2: Effective decay exponent $\alpha(t)$ for the two-dimensional $q$-CAM with $q=2,3,4$, and 5 (random initial conditions, equal initial particle numbers for each species). The data were obtained on a $1000 \times 1000$ square lattice, averaged over 20 runs.

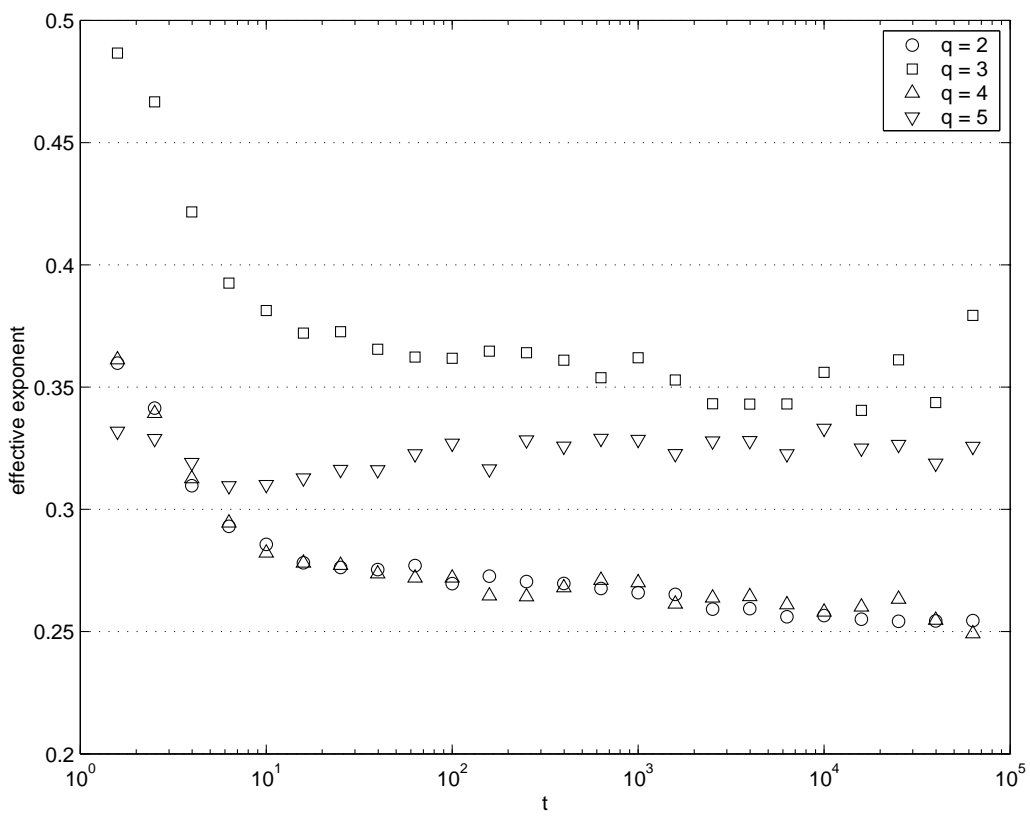

Figure 3: Effective decay exponent $\alpha(t)$ for the one-dimensional $q$-CAM with $q=2,3,4$, and 5 (random initial conditions, equal initial particle numbers for each species). The data were obtained on a $10^{5}$ lattice, averaged over 20 runs. 
dimension given in Eq. (29).

In summary, our simulation results for the $q$-CAM, within the accuracy and statistical errors of our data, are consistent with the analytical predictions of the previous sections, if we allow for crossover effects induced by the presence of the competing mean-field decay law. The equivalence of the 4-CAM and 2-CAM, which are both identical to the 2-MAM $(A+B \rightarrow 0)$, is clearly demonstrated. Since the 3-CAM and 3-MAM are identical, only our data for $q=5$ yield novel results. Unambiguous identification of the density exponents $\alpha$ would ideally require better statistics, that is, simulations on considerably larger systems.

\section{Differential equation approach}

Before concluding we wish to make a methodological remark. One may attempt to address the issue of segregation by considering space-dependent particle densities $\rho_{i}(x, \tau)$ that satisfy the reaction-diffusion equations (with diffusivity $D$ )

$$
\frac{\partial \rho_{i}(x, \tau)}{\partial \tau}=D \Delta \rho_{i}-\sum_{j} \kappa_{i j} \rho_{i} \rho_{j} .
$$

For spatially uniform (well-mixed) systems these reduce to the ordinary differential equations (41). Let us now apply to the system (33) what is essentially a linear stability analysis.

To this end, we write $\rho_{i}(x, \tau)=\rho(\tau)\left[1+\epsilon_{i}(x, \tau)\right]$ and linearize with respect to the perturbations $\epsilon_{i}$. With Eqs. (44) this yields

$$
\frac{\partial \epsilon_{i}(x, \tau)}{\partial \tau}=D \Delta \epsilon_{i}(x, \tau)-\rho(\tau) \sum_{j} \kappa_{i j} \epsilon_{j},
$$

where the neglected nonlinear terms are of order $\rho \epsilon^{2}$. In terms of the linearly transformed variables

$$
\hat{\epsilon}_{\mu}(p, \tau)=\sum_{j} U_{\mu j} \int \mathrm{d}^{d} x \mathrm{e}^{\mathrm{i} p \cdot x} \epsilon_{j}(x, \tau)
$$

with $U$ as defined in Subsec. 3.3. the time evolution equation (34) becomes diagonal and reads

$$
\frac{\mathrm{d} \hat{\epsilon}_{\mu}(p, \tau)}{\mathrm{d} \tau}=-\left[D p^{2}+\rho(\tau) \hat{\kappa}_{\mu}\right] \epsilon_{\mu}(p, \tau) .
$$

For given initial $\hat{\epsilon}_{\mu}(p, 0)$ this is solved by

$$
\hat{\epsilon}_{\mu}(p, \tau)=\hat{\epsilon}_{\mu}(p, 0)(1+\tau)^{-\hat{\kappa}_{\mu}} \mathrm{e}^{-D p^{2} \tau} .
$$

Irrespective of the value of $\hat{\kappa}_{\mu}$ this solution exists and tends to zero as $\tau \rightarrow \infty$. Nevertheless, for $\hat{\kappa}_{\mu}<-D p^{2}$ it will increase initially. In the event that $\left|\hat{\epsilon}_{\mu}\right|$ grows with time such as to be no longer negligible with respect to unity at some instant of time, this signals that the neglected nonlinear terms in the differential equation begin to play a role; in particular, they will prevent $\epsilon_{i}$ from decreasing beyond -1 and hence $\rho_{i}$ from turning negative. We will argue, however, that in this case the physical justification for the differential equations (33) breaks down, and that in fact segregation sets in. 
This goal requires going beyond the mathematics and invoking the interpretation of the density as an average over discrete particles. For particles initially randomly and uniformly distributed in a volume $L^{d}$, we typically have that $\hat{\epsilon}_{\mu}(p, 0) \sim L^{-d / 2}$. If this initial fluctuation is negative, and if in the course of time $\hat{\epsilon}_{\mu}(p, \tau)$ approaches -1 , this should be interpreted properly as there being an appreciable probability for species extinction. A necessary condition for the r.h.s. of Eq. (37) to become of order unity reads explicitly $L^{-d / 2}(1+\tau)^{-\hat{\kappa}_{\mu}} \gtrsim 1$, or, since $\hat{\kappa}_{\mu}$ must be negative, $\tau \gtrsim \tau_{L} \equiv L^{d /\left|2 \hat{\kappa}_{\mu}\right|}$. But on these time scales $D p^{2} \tau$ must still be much less than unity. Taking the smallest allowed valued for $p$ in the volume $L^{d}$, that is, $p_{L} \sim 2 \pi / L$, the additional condition $D p_{L} \tau_{L} \ll 1$ yields $L^{d /\left|2 \hat{\kappa}_{\mu}\right|-2} \ll 1$, which will happen when $d<4\left|\hat{\kappa}_{\mu}\right|$.

Hence the differential equation approach, combined with appropriate considerations in which the particle discreteness intervenes, leads to the identical expression for the critical segregation dimension as found in Eq. (21) of Sec. 5. The differential equation approach was followed, essentially, in Ref. [31]. To our opinion, however, the Fokker-Planck method used in Refs. [28, 33] and in the present work is preferable, since it is based directly on the more fundamental description of an interacting many-body problem in terms of a master equation. More specifically, the stochasticity taken into account in the differential equation approach is due only to the random fluctuations present in the initial state. As a consequence, this approach wrongly suggests that the initially dominant species (or, more precisely, their initially dominant mode $\mu$ ) is also the surviving one.

It follows from the Fokker-Planck equation that there is actually an interplay between initial and dynamically generated fluctuations, brought out by the solution (13) of the second moment equations. This solution contains a dynamically generated contribution to the fluctuations (viz. the terms $\propto K_{\mu}$ ), and a contribution due to the initial fluctuations (the $\Gamma_{0}$ term). In systems without segregation (i.e., when $\hat{\kappa}_{\mu} \geq-\frac{1}{2}$ ) the dynamically generated fluctuations become larger than those due to the initial conditions, which are eventually forgotten. In systems with segregation (for $\hat{\kappa}_{\mu}<-\frac{1}{2}$ ) the initial and dynamical contributions are of the same order and the initial conditions co-determine the final state. In this weaker sense, the segregation phenomenon that appears in the cases with $\hat{\kappa}_{\mu}<-\frac{1}{2}$ is still linked to the persistence of initial fluctuations, even in the absence of conservation laws.

\section{Conclusion}

We have studied a wide class of $q$-species reaction-diffusion systems with pair annihilation processes between distinct species. This class includes the two well-studied paradigmatic cases $A+A \rightarrow 0$ (in the limit $q \rightarrow \infty)$ and $A+B \rightarrow 0(q=2)$. Within mean-field theory, i.e. for spatial dimensions $d \geq 2$, we have determined for each member of the class (i) whether or not segregation occurs, and (ii) the value of the decay exponent $\alpha$ in the asymptotic power law for the total particle density. Our findings represent a considerable extension of previous work on segregation in diffusion-limited annihilation reactions. Our preliminary simulation data are compatible with the analytical results.

Our method builds on ideas that were applied earlier in the context of $q$-species models by Ben-Avraham and Redner [28, and more recently also by Ben-Naim and Krapivsky 
33. and Newman and McKane [34. This approach is obviously not limited to the special cases studied here, but we have not aimed at being exhaustive. It is straightforward, for example, to drop the restriction of no self-annihilation $\left(k_{i i}=0\right)$, and redo the analysis. The method may also be employed to analyze more involved situations, such as models with species belonging to two distinct equivalence classes.

Various open problems remain, in particular concerning the nature of the domain structure in several important cases. Furthermore, the theory presented here is unable to address the issue of what happens in low dimensions, $d<2$, where particle anticorrelations become manifest and render the mean-field treatment invalid. We expect that more elaborate simulations will be carried out in the future to test our predictions as well as to study questions beyond reach of the present theory.

\section{Acknowledgements}

This material is based upon work supported by the National Science Foundation, Division of Materials Research, under Grant No. DMR-0308548. The Laboratoire de Physique Théorique of the Université de Paris-Sud is associated with the Centre National de la Recherche Scientifique as research unit UMR 8627.

\section{References}

[1] M. von Smoluchoswki, Z. Phys. 17, 557 (1916).

[2] M. Bramson and D. Griffeath, Ann. Prob. 8, 183 (1980) [Z. Wahrsch. Verw. Gebiete 53, $183(1980)]$.

[3] A. A. Ovchinnikov and Ya. B. Zeldovich, Chem. Phys. 28, 215 (1978).

[4] M. Droz, Z. Rácz, and J. Schmidt, Phys. Rev. A 39, 2141 (1989).

[5] L. Peliti, J. Phys. A 19, L365 (1986).

[6] K. Kroon, H. Fleurent, and R. Sprik, Phys. Rev. E 47, 2462 (1993).

[7] D. Toussaint and F. Wilczek, J. Chem. Phys. 78, 2642 (1983).

[8] E. Monson and R. Kopelman, Phys. Rev. E 69, 021103 (2004).

[9] K. Kang and S. Redner, Phys. Rev. Lett. 52, 955 (1984).

[10] F. Leyvraz and S. Redner, Phys. Rev. Lett. 66, 2168 (1991); Phys. Rev. A 46, 3132 (1992).

[11] M. Bramson and J. L. Lebowitz, Phys. Rev. Lett. 61, 2397 (1988); J. Stat. Phys. 62, 297 (1991); ibid. 65, 941 (1991).

[12] B. P. Lee, J. Phys. A 27, 2633 (1994).

[13] B. P. Lee and J. Cardy, J. Stat. Phys. 80, 971 (1995); ibid. 87, 951 (1997). 
[14] K. Oerding, J. Phys. A 29, 7051 (1996).

[15] V. Kuzovkov and E. Kotomin Rep. Prog. Phys. 51, 1479 (1988).

[16] A. A. Ovchinnikov, S. F. Timashev, and A. A. Belyy, Kinetics of Diffusion-Controlled Chemical Processes (Nova Science, New York, 1989).

[17] F.C. Alcaraz, M. Droz, M. Henkel, and V. Rittenberg, Ann. Phys. (NY) 230, 250 (1994).

[18] M. Henkel, E. Orlandini, and J. Santos, Ann. Phys. (NY) 259, 163 (1997).

[19] V. Privman, Ed., Nonequilibrium statistical mechanics in one dimension, Cambridge University Press (1997).

[20] J. Cardy, in Proceedings of Mathematical Beauty in Physics, ed. J.-B. Zuber, Adv. Ser. Math. Phys. Vol. 24 (World Scientific, River Edge, NJ, 1997), p. 113.

[21] D. C. Mattis and M. L. Glasser, Rev. Mod. Phys. 70, 979 (1998).

[22] B. Chopard and M. Droz, Cellular Automaton Modeling of Physical Systems (Cambridge University Press, Cambridge, 1998).

[23] J. Marro and R. Dickman, Nonequilibrium Phase Transitions in Lattice Models (Cambridge University Press, Cambridge, 1999).

[24] H. Hinrichsen, Adv. Phys. 49, 815 (2000).

[25] G. M. Schütz, in Phase Transitions and Critical Phenomena, ed. C. Domb and J. L. Lebowitz, Vol. 19 (Academic Press, London, 2001).

[26] G. Ódor, Rev. Mod. Phys. 76, 663 (2004).

[27] M. Howard, U. C. Täuber, and B. P. Vollmayr-Lee, to appear in J. Phys. A (2004).

[28] D. Ben-Avraham and S. Redner, Phys. Rev. A 34, 501 (1986).

[29] O. Deloubrière, H. J. Hilhorst, and U. C. Täuber, Phys. Rev. Lett. 89, 250601 (2002).

[30] D. Zhong, R. Dawkins, and D. Ben-Avraham, Phys. Rev. E 67, 040101 (2003).

[31] H. J. Hilhorst, O. Deloubrière, M. J. Washenberger, and U. C. Täuber, J. Phys. A 37, 7063 (2004).

[32] N. G. van Kampen, Stochastic Processes in Physics and Chemistry (North-Holland, Amsterdam 1992).

[33] E. Ben-Naim and P. L. Krapivsky, Pys. Rev. E 69, 046113 (2004).

[34] T. Newman and A. McKane, Preprint (2004). 